\title{
ON THE CONNECTIFICATION OF A SPACE BY A COUNTABLE POINT SET
}

\author{
GARY MILLER and B. J. PEARSON \\ (Received 30 January 1969; revised 14 October 1969) \\ Communicated by B. Mond
}

In this note we define a rather pathological connectedness property of Hausdorff spaces which is stronger than ordinary connectedness. We obtain a few basic properties of such spaces and derive a method for constructing them. It turns out that countable Hausdorff spaces having the connectedness property are as easily constructed as uncountable ones. Hence we have still another method for constructing countable connected Hausdorff spaces.

Definition. The subset $K$ of the space $X$ saturates $X$ if $K$ is infinite and for each open set $U$ in $X, K-\bar{U}$ is finite. The space $X$ is said to be saturated if $X$ is a point or some subset of $X$ saturates $X$.

THEOREM 1. If $U$ is an open subset of the saturated space $X$, then $\bar{U}$ is connected.

Proof. Suppose $K$ is a subset of $X$ that saturates $X$ and $U$ is an open subset of $X$ such that $\bar{U}$ is not connected. Then $\bar{U}$ is the union of two disjoint closed sets $R$ and $S . U-S$ is open, $\overline{U-S} \subseteq R$, and hence $K-R$ is finite. Similarly, $K-S$ is finite. But $K=(K-R) \cup(K-S)$.

COROLlary. Every saturated space is connected.

THEOREM 2. If $x$ and $y$ are two points of the saturated space $X$ and $\mathscr{S}$ is an open cover of $X$, then there is a simple chain of elements of $\mathscr{S}$ from $x$ to $y$ with at most three links.

Proof. Suppose $x \in U \in \mathscr{S}$ and $y \in W \in \mathscr{S}$. Since $\overline{U \cup W}$ is connected, there is a point $z$ such that $z \in \bar{U} \cap \bar{W}$. Let $V$ be an element of $\mathscr{S}$ containing $z$, and form the simple chain from the sets $U, V$, and $W$.

THEOREM 3. If $X$ is saturated, then no finite subset of $X$ separates $X$.

Proof. Suppose $K$ saturates $X, H$ is a finite subset of $X$, and $X-H$ is the union of two disjoint open sets $U$ and $V$. But then $K$ is the union of the three finite sets, $K-\bar{U}, K-\bar{V}$, and $K \cap H$. 
THEOREM 4. Iff is a continuous map of the saturated space X onto the Hausdorff space $Y$, then $Y$ is saturated.

Proof. Suppose $K$ saturates $X$. For each open set $U$ in $\left.Y, \overline{f\left(f^{-1}(U)\right.}\right) \subseteq$ $f\left(f^{-1}(\bar{U})\right)=\bar{U}$ and hence $f\left(K-\overline{f^{-1}(U)}\right) \supseteq f(K)-\bar{U}$. Since $K-\overline{f^{-1}(U)}$ is finite, $f(K)-\bar{U}$ is finite. It follows that if $f(K)$ is infinite, it saturates $Y$. Suppose $f(K)$ is finite. Let $\left\{U_{1}, \cdots, U_{n}\right\}$ be a collection of disjoint open sets in $Y$ covering $f(K)$. For some $i, K \cap f^{-1}\left(U_{i}\right)$ is infinite, and hence if $j \neq i, K-\overline{f^{-1}\left(U_{j}\right)}$ is infinite. It follows that $f(K)$ is a point $y$ of $Y$. Finally, suppose that $Y$ contains a point $z$ different from $y$. Let $U$ and $V$ be two disjoint open sets containing $y$ and $z$ respectively. Then $K \subseteq f^{-1}(U)$ and hence $K-\overline{f^{-1}(V)}$ is infinite. Thus it has been shown that either $f(K)$ is infinite and saturates $Y$ or $Y$ consists of a single point.

\section{COROLlaRY. A continuous map of a saturated space into $E^{1}$ is a constant map.}

THEOREM 5. Every separable metric space $X$ is a subspace of a saturated completely separable locally metrizable Hausdorff space $Z$ such that $Z-X$ is countable.

Proof. Let $H$ denote Hilbert space, and let $H^{\prime}$ denote the set of all points $\left(x_{1}, x_{2}, \cdots\right)$ of $H$ such that $x_{1}=0$. Let $X$ be embedded as a subset of $H^{\prime}$. In what follows, an $\varepsilon$-net of the metric space $M$ is a subset $N$ of $M$ such that each point of $M$ is within a distance of $\varepsilon$ of some point of $N$, and the mesh of $N$, denoted by $|N|$, is defined by $|N|=\inf \{d(x, y) \mid x, y \in N$ and $x \neq y\}$. For each $i$, let $\varepsilon_{i}=1 / 2^{i}$. Clearly, there exists a sequence $N_{1}, N_{2}, \cdots$ of subsets of $H-H^{\prime}$ such that for each $i, N_{i}$ is an $\varepsilon_{i}$-net of $H-H^{\prime},\left|N_{i}\right| \geqq \varepsilon_{i}$, and $N_{i} \subset N_{i+1}$. Since $H^{\prime}$ is the boundary of $H-H^{\prime}$, each $N_{i}$ is an $\varepsilon_{i}$-net of $H$. Let $w_{1}=N_{1}$, and for each $i>1$, let $w_{i}=N_{i}-N_{i-1}$. It is easily seen that for each $i, w_{i}$ is a $4 \varepsilon_{i}$-net of $H,\left|w_{i}\right| \geqq \varepsilon_{i}$, and if $j \neq i, d\left(w_{i}, w_{j}\right)>0$. Since each $w_{i}$ is countable, there exists a countable subset $K$ of $\left(H-H^{\prime}\right)-\bigcup_{i=1}^{\infty} w_{i}$ such that $K$ is dense in $H$. Let $W=\bigcup_{i=1}^{\infty}\left\{w_{i}\right\}$, let $Y=X \cup K$, and let $Z=W \cup Y$.

We now define a topology for $Z$. For each $i$ and each point $x$ of $w_{b}$, let $U_{i 1}^{x}, U_{i 2}^{x}, \cdots$ be a sequence of spherical neighborhoods of $x$ in $H$ such that for each $n, U_{i n}^{x}$ has radius $\varepsilon_{i} / 3 n$. For each $i$ and $n$, let $U_{i n}=\bigcup\left\{U_{i n}^{x} \mid x \in w_{i}\right\}$ and let $V_{\text {in }}=\left(U_{\text {in }} \cap Y\right) \cup\left\{w_{i}\right\}$. Let $\mathscr{R}$ denote the relative topology of $Y$ in $H$, let $\mathscr{C}$ denote the collection of all such sets $V_{i n}$, and let $\mathscr{R}=\mathscr{R} \cup \mathscr{C}$. It is easily seen that $\mathscr{B}$ is closed with respect to set intersection, so that $\mathscr{B}$ induces a topology $\mathscr{I}$ on $Z$. Also, since $\mathscr{R}$ has a countable base and $\mathscr{C}$ is countable, some countable subcollection of $\mathscr{B}$ induces $\mathscr{I}$. In what follows, $Z$ is considered as a space with topology $\mathscr{I}$.

Suppose $x$ and $y$ are distinct points of $Z$. Clearly, if $x, y \in Y$, there are disjoint open sets in $Z$ containing $x$ and $y$ respectively. Suppose $x \in Y$ and for some $i$, $y=w_{i}$. There exists an $n$ such that $\varepsilon_{i} / 3 n<d\left(x, w_{i}\right)$. Hence $\left(H-\overline{U_{i n}}\right) \cap Y$ and $V_{i n}$ are disjoint open sets in $Z$ containing $x$ and $y$ respectively. Suppose $x=w_{i}$ and $y=w_{j}$. Let $\varepsilon=d\left(w_{i}, w_{j}\right)$. There exists an $n$ such that $\left(\varepsilon_{i}+\varepsilon_{j}\right) / 3 n<\varepsilon / 2$. Hence 
$V_{i n}$ and $V_{j n}$ are disjoint open sets in $Z$ containing $x$ and $y$ respectively. Therefore $Z$ is a Hausdorff space.

Suppose $U$ is an open set in $Z$. Let $p \in U \cap Y$, and let $S$ be an $\varepsilon$-neighborhood of $p$ in $H$ such that $S \cap Y \subseteq U$. There exists a $k$ such that $\varepsilon_{k}<\varepsilon / 4$. Hence for each $i>k, w_{i} \cap S \neq \emptyset$. Suppose $i>k$ and $V$ is an open set in $Z$ containing $w_{i}$. There exists an $n$ such that $V_{i n} \subseteq V$ and for some point $x$ of $S \cap w_{i}, U_{i n}^{x} \subseteq S$. Since $K$ is dense in $H, U_{i n}^{x}$ contains a point $y$ of $K$. Hence $y \in V \cap U$ and $y \neq w_{i}$. We have thus shown that for each open set $U$ in $Z$ there exists a $k$ such that if $i>k$, then $w_{i}$ is a limit point of $U$. It follows that $W$ saturates $Z$.

It remains to be shown that $Z$ is locally metrizable. If $U \subseteq S \subseteq H, \mathrm{Cl}_{S}(U)$ denotes the closure of $U$ in the subspace $S$ of $H$. For each $i$, let $Y_{i}$ denote the subspace $Y \cup\left\{w_{i}\right\}$ of $Z$. Since the relative topology of $W$ is discrete, $Y_{i}$ is open in $Z$. Furthermore, the relative topology of $Y$ in $Y_{i}$ is the same as the relative topology of $Y$ in $H$. It follows that $Y$ is a regular subspace of $Y_{i}$. Suppose $V$ is an open set in $Y_{i}$ containing $w_{i} . V$ is open in $Z$. Hence there exists an $n$ such that $V_{i n} \subseteq V$. Let $m>n$. Since $\mathrm{Cl}_{H}\left(U_{i m}\right) \subseteq U_{i n}, \mathrm{Cl}_{Y_{i}}\left(V_{i m}\right) \subseteq V$. Therefore $Y_{i}$ is regular at $w_{i}$. Suppose $x \in Y$ and $U$ is an open set in $Y_{i}$ containing $x$. There is an open set $V_{1}$ in $Y$ containing $x$ such that $\mathrm{Cl}_{Y}\left(V_{1}\right) \subseteq U . \mathrm{Cl}_{Y}\left(V_{1}\right)=\mathrm{Cl}_{Y_{i}}\left(V_{1}\right)-\left\{w_{i}\right\}$. Since $Y_{i}$ is Hausdorff, there exists an open set $V_{2}$ in $Y_{i}$ containing $x$ such that $w_{i} \notin \mathrm{Cl}_{Y_{i}}\left(V_{2}\right)$. Let $V=V_{1} \cap V_{2}$. Then $\mathrm{Cl}_{Y_{i}}(V) \subseteq \mathrm{Cl}_{Y_{i}}\left(V_{1}\right) \cap \mathrm{Cl}_{Y_{i}}\left(V_{2}\right) \subseteq \mathrm{Cl}_{Y_{i}}\left(V_{1}\right)-\left\{w_{i}\right\}=$ $\mathrm{Cl}_{Y}\left(V_{1}\right) \subseteq U$. Therefore $Y_{i}$ is regular, and since $Y_{i}$ is also completely separable, it is metrizable. Thus $Z=\bigcup_{i=1}^{\infty} Y_{i}$, and for each $i, Y_{i}$ is an open metrizable subspace of $Z$. It follows that $Z$ is locally metrizable. This completes the proof of the theorem.

THEOREM 6. Every countable metric space $S$ which is dense in itself is a subspace of a countable completely separable locally metrizable Hausdorff space $Z$ such that $Z-S$ is closed, discrete, and saturates $Z$.

Indication of Proof. In the proof of Theorem 5, let $X$ be empty. $Y$ is then a countable subset of Hilbert space homeomorphic to $S$. In fact, $E^{n}$ for any $n \geqq 1$ may be used in place of Hilbert space.

From Theorem 6, we obtain an example of a nondegenerate countable connected Hausdorff space. Of the other known examples, the one which seems to resemble ours the most is the one given by Bing [1]. It is not hard to see that in Bing's example there exist three open sets $U, V$, and $W$ such that $\bar{U} \cap \bar{V} \cap \bar{W}=\emptyset$. It follows that Bing's example is not saturated.

\section{References}

[1] R. H. Bing, 'A countable connected Hausdorff space', Proc. Amer. Math. Soc. 59 (1953), 474.

[2] M. Brown, 'A countable connected Hausdorff space', Bull. Amer. Math. Soc. 59 (1953), 367.

[3] E. Hewitt, 'On two problems of Urysohn', Annals of Mathematics, 47 (1946), 503-509. 
[4] J. Martin, 'A countable connected Hausdorff space with a dispersion point', Duke Math. J. 33 (1966), 165-167.

[5] P. Urysohn, 'Über die Mächtigkeit der Zusammenhängen Mengen', Mathematische Annalen, 94 (1925), 262-295.

University of Missouri at Kansas City 